\title{
Evaluating the Performance of Modified Watershed Algorithm Based on DBMF and DT
}

\author{
Inderpal Singh ${ }^{1}$, Dinesh Kumar ${ }^{2}$
}

Research Scholar in Computer Science and Engineering Department, DAV Institute of Engineering and Technology Jalandhar (Punjab), India,

Er.inderpal13@gmail.com ${ }^{1}$

Faculty of IT Department

DAV Institute of Engineering and Technology Jalandhar (Punjab), India, dinesh_daviet@hotmail.com²

\section{ABSTRACT}

The image segmentation; segments a given image into separate regions and objects. It is widely used in various vision applications like face detections, motion detection etc. It has been found that the noise has affected the segmentation at a great extent; so to overcome this problem we have presented a modified watershed segmentation using Decision based median filtering (DBMF) and Dynamic thresholding (DT). DBMF has the ability to reduce the high density of noise in the image so will provide more efficient results. However DT has also refined segmentation process further. The comparisons have shown that the proposed algorithm provides quite significant results over the available methods.

\section{Indexing terms/Keywords}

Image Segmentation, Watershed, Clustering, THRESHOlding.

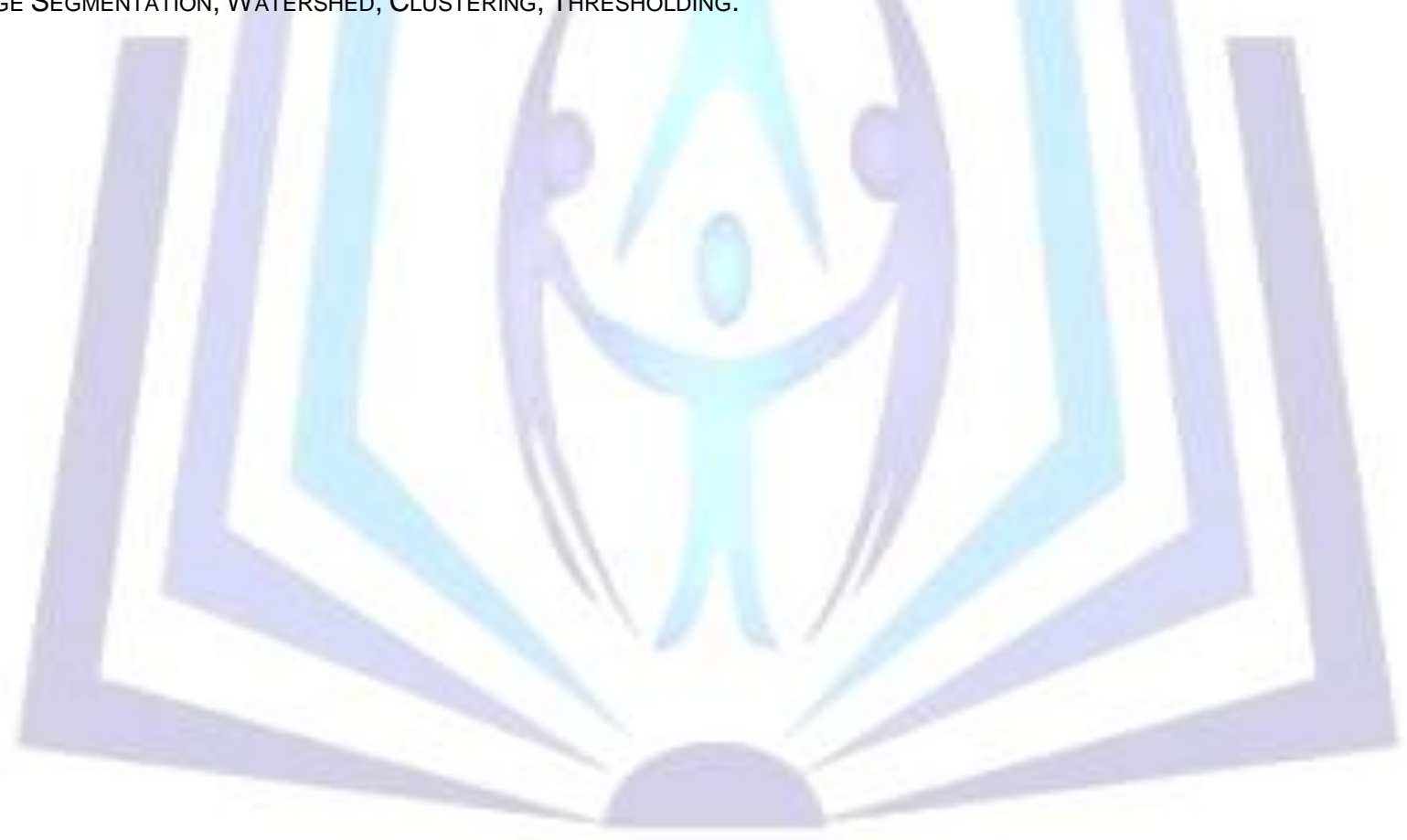

\section{Council for Innovative Research}

Peer Review Research Publishing System

Journal: INTERNATION JOURNAL OF COMPUTERS AND TECHNOLOGY

Vol. 13 , No. 4

editorijctonline@gmail.com

www.cirworld.org/journals 


\section{INTRODUCTION}

Image segmentation is vital step in the process of image processing. Image segmentation is to divide an image into number of regions so that each region gives information about an object or area of interest [1]. It partitions an image into a set of non-overlapping regions whose union is the entire image. Watershed is an algorithm for image segmentation developed in recent years based on mathematical morphology [1], and has draw great attention for its fast computing and high accuracy in locating the weak edges of adjacent regions.

But classical watershed segmentation is sensitive to noise and can leads to serious over-segmentation. Therefore, many researchers has proposed various methods continually for improving, such as a preprocessing step of calculating distance transform for the binary image before watershed transformation [2], or agglomerating regions after segmentation following watershed transformation [3].

In this paper, we propose a modified watershed algorithm to reduce over- segmentation that uses both pre- and postprocessing. We make use of more prior knowledge in pre-processing and merge redundant minimal regions in the postprocessing.

\section{WATERSHED TRANFORM}

Watershed transform has concerned with great attention in recent years as an efficient morphological image segmentation tool. It is similar to region-based approach; it begins the growing process from every regional minimum point, each of which creates a single region after the transform. Watershed algorithm combines both the discontinuity and similarity properties successfully [5].

It performs well when it can distinguish the background location and the foreground object. It is based on grayscale mathematical morphology. The main drawback of watershed transform is over-segmentation, sensitive to noise and high computational complexity those make it unsuitable for real-time process [6].

\section{RELATED RESEARCH}

The watershed transformation is a powerful tool for image segmentation based on well-known mathematical morphologybased approach. In reference [7], the authors propose an improved method watershed algorithm for natural color image segmentation based on adaptive marker-extraction to overcome over-segmentation of the watershed transform.

In reference [8], the authors have presented a marked extraction based on adaptive color image segmentation algorithm to improve the watershed, the traditional marker for the lack of extraction methods, many consider the minimum characteristics of properties, and set the adaptive threshold. The authors proposed method performs better than original watershed algorithm with a strong anti-noise performance.

In reference [3], the authors presented an integrated K-Means clustering algorithm with marker controlled watershed segmentation algorithm. Several approaches exist to solve the over- segmentation problem, such as integrating watershed with region merging algorithm [9], watershed based on gradient modification and hierarchical region merging algorithms [10], combined marker-based watershed and region merger [11], marker-controlled watershed crown segmentation [12], morphological gradient applied to new active contour model [13], marker-based watershed algorithm [14], and interactive segmentation by matching attributed relational graphs are based on watershed, graph cuts, shortest paths (geodesic) and random walker [15].

\section{PROPOSED MODIFIED WATERSHED ALGORITHM}

This research work focuses on the image segmentation using masking or marker based watershed segmentation. This research work improves the segmentation by integrating the decision based switching median filter with masking based watershed segmentation to enhance the results further. The decision based switching median filter will be used as preprocessing operation to reduce the effect of the noise, haze, fog etc. to improve the marking process for watershed based segmentation we will use dynamic thresholding to mark the objects in efficient manner.

The image gradient will also be used to find the edges of the image in the efficient manner. This will help the proposed algorithm to segment the local object in efficient manner, as edges will be known in prior using gradient smoothing. 


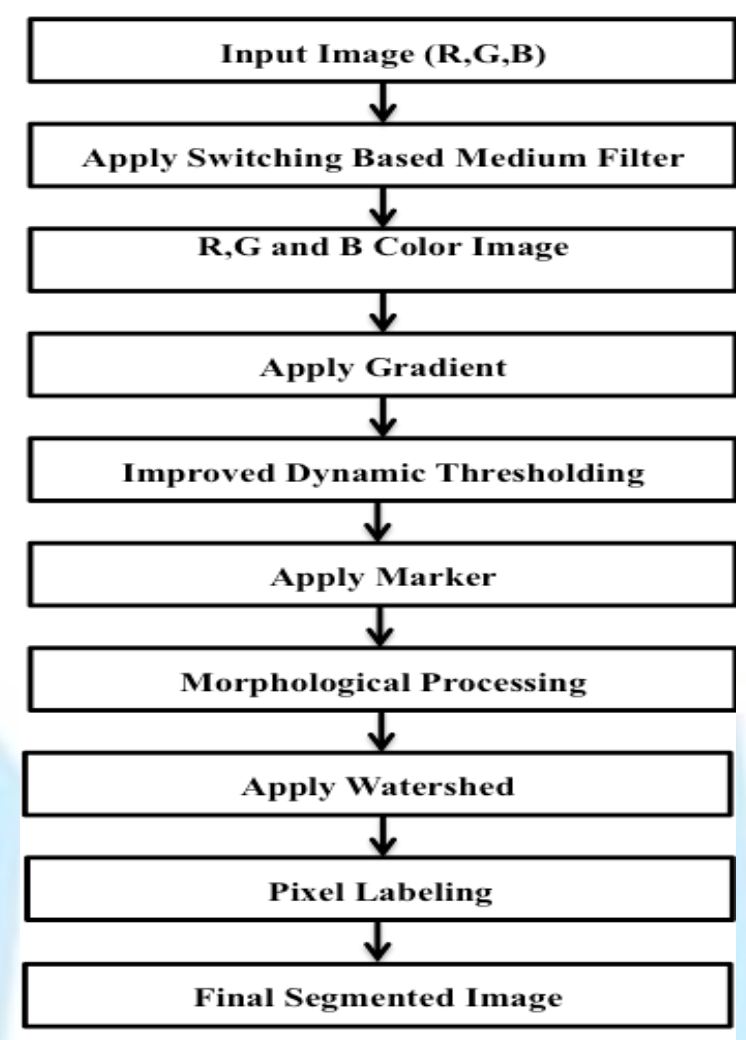

Figure 1.0: The flow chart of the proposed modified watershed algorithm

\section{Result And Discussion}

We tested the offered algorithm on images taken from the Berkeley image database [25]. The algorithms are implemented in MATLAB code and tested on a notebook (Intel (R) Core (TM) i7-2670QM 2.20GHz CPU, 4GB Memory, Windows 7 OS), using a quad core for the processing.

Step 1.Input Image: An image passed to the proposed algorithm. Image must be either 2-D or 3-D plane i.e. grey scale or color image.

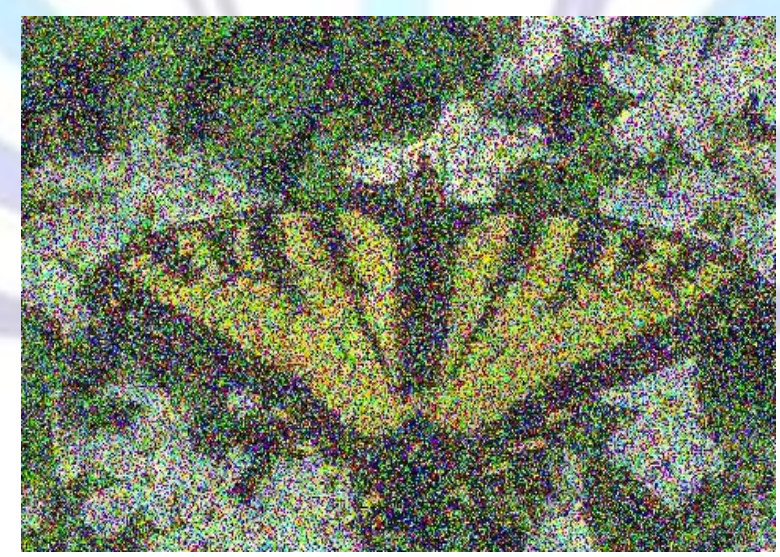

Figure 1.1: Input Image

Step 2.Apply Switching based Medium Filter: It is used to restore images corrupted by salt-pepper impulse noise. The salt \& pepper noise is a special type of impulse noise in which some portion of image pixel values are replaced by either minimum or maximum pixel values.

The main objective of salt \& pepper noise removal is that it removes the noise from the image by preserving the other image details. 


$$
P^{\prime}(m, \pi, k)=\frac{1}{b} \sum_{i=-s}^{s} \sum_{i=-s}^{s} \rho(m-i, n-j, k)
$$

Where (2S I 1j L (Lis order of the window e.g. 3,5,7), P(m i, $\mathbf{l} \quad \mathrm{j}, \mathrm{k}$ ) is a healthy pixel in the window, if not ),

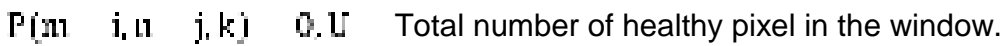

We have used an RGB image as input color image. The original image is extracted into individual red (R), green $(G)$, and blue (B) color channels as shown Figure 1.3.

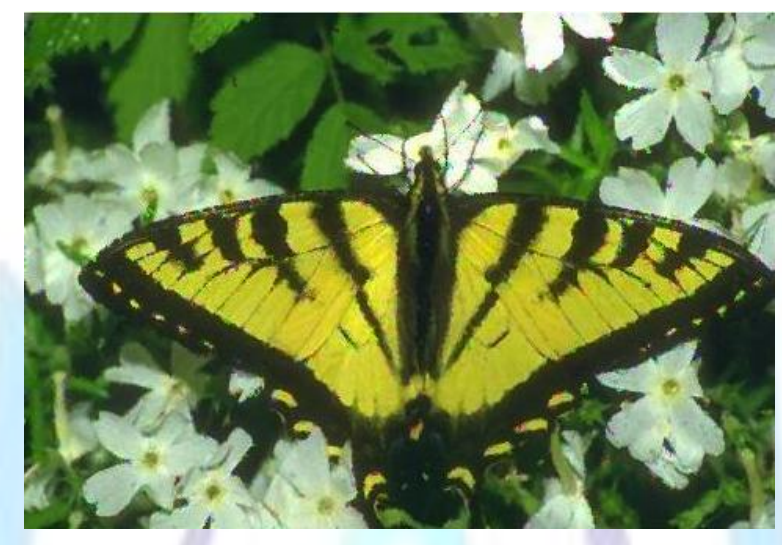

Figure 1.2: Result of Improved Medium Filter

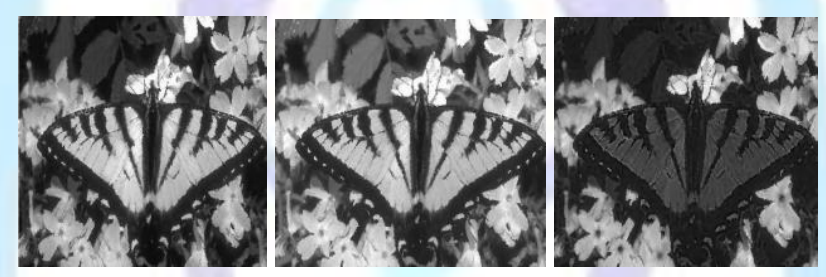

Figure 1.3: Extracted Original image into R, G and B channels

Step 3.Apply Gradient: Mathematically the gradient of a two-variable function at each image point is a 2D vector with the components given by the derivatives in the horizontal and vertical directions. At each image point, the gradient vector points in the direction of largest possible intensity increase, and the length of the gradient vector corresponds to the rate of change in that direction.

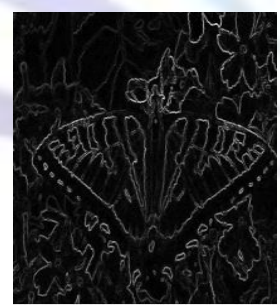

Figure 1.4:(a)

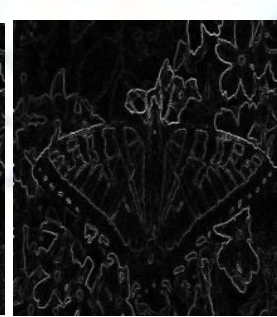

Figure 1.4:(b)

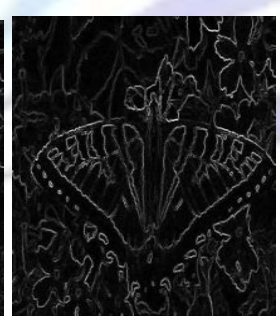

Figure 1.4:(c)

Figure 1.4:(a) Red Channel Gradient Magnitude Figure 1.4:(b) Green Channel Gradient Magnitude Figure 1.4:(c) Blue Channel Gradient Magnitude

Step 4.Improved Dynamic Thresholding: Thresholding is used to segment an image by setting all pixels whose intensity values are above a threshold to a foreground value and all the remaining pixels to a background value. Whereas the conventional thresholding operator uses a global threshold for all pixels, adaptive thresholding changes the threshold dynamically over the image. 


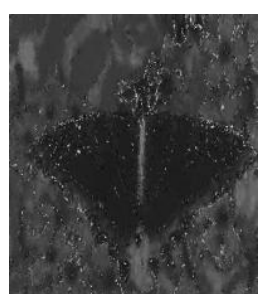

Figure 1.5:(a)

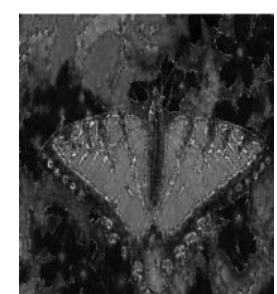

Figure 1.5:(b)

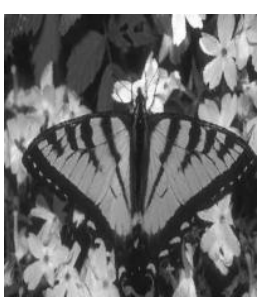

Figure 1.5:(c)

Figure 1.5:(a) Dynamic Thresholding of Red Channel Figure 1.5:(b) Dynamic Thresholding of Green Channel Figure 1.5:(c) Dynamic Thresholding of Blue Channel

Step 5.Apply Marker: Markers can be used to solve the over-segmentation problem whose goal is to detect the presence of homogeneous regions from the image by a set of morphological simplifications. The watershed transform is often applied to this problem. Segmentation using the watershed transform works better if you can identify, or "mark," foreground objects and background locations.

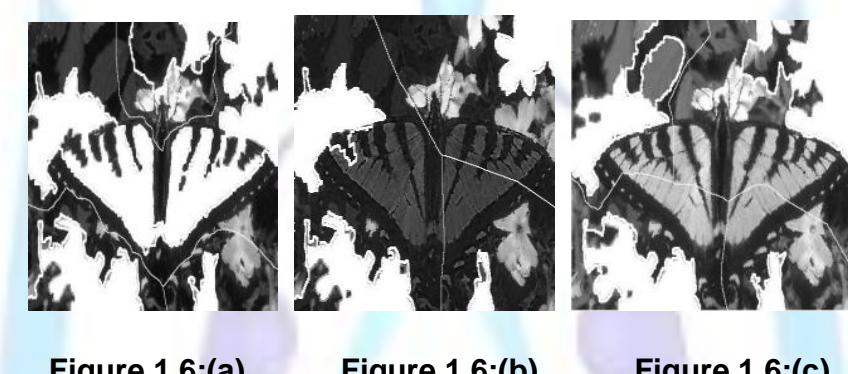

Figure 1.6:(a) Red Channel Markers and object boundaries Figure 1.6:(b) Green Channel Markers and object boundaries Figure 1.6:(c) Blue Channel Markers and object boundaries

Step 6. Morphology Processing: Mathematical morphology as a tool for extracting image components that are useful in the representation and description of region shape, such as boundaries, skeletons, etc. We assume that function $f(x)$ can express gray image I, $x$ define gray value in pixels. Structuring element B denotes as structure operator. Its shape can be described by S, with size $n^{*} n$. morphology dilation can be defined as follows:

$$
\begin{aligned}
& f(x) \quad \operatorname{kax}(f(x) \text { kj) } k \subset B \\
& f(x) \quad \text { Wax }(f(x) k j) \quad k \subset B
\end{aligned}
$$

Step 7. Apply Watershed: The Final Gradient image, which is marker, extracted is subjected to Watershed Segmentation.

Let $f \subset C(D)$ have minima $\left.m_{\mathrm{L}}\right]_{\mathrm{k}, \mathrm{r}}$ for some index set I. The catchment basin $C B\left(m_{4}\right)$ of a minimum mi is defined as the set of points $x \subset D$ which are topographically closer to mj than to any other regional minimum $\mathrm{mj}$ :

$$
\text { CB }\left(m _ { 1 } j \quad \left(x \in D|\Psi| T(i): f\left(m_{1} j\left|T_{1}\left(x_{1} m_{1}\right)<f\left(m_{1}\right)\right| T_{r}\left(x_{2} m_{i}\right)\right.\right.\right.
$$

The watershed of $f$ is the set of points, which do not belong to any catchment basin:

$$
\text { wuiersited }(\delta)=D n\left(:=C D\left(m_{1}\right)\right)
$$

Let $\mathrm{W}$ be some label, $W \subset I$ The watershed transform of $\mathrm{f}$ is a mapping $\lambda: D, I \cup\{w\}$ such that:

Alpj i if pecB(n)

And

dipj lwif peratersied $(f)$ 
So the watershed transform of $f$ assigns labels to the points of $D$, such that (I) different catchment basins are uniquely labeled, and (II) a special label $\mathrm{W}$ is assigned to all points of the watershed of $\mathrm{f}$.

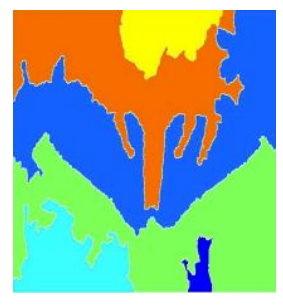

Figure 1.7:(a)

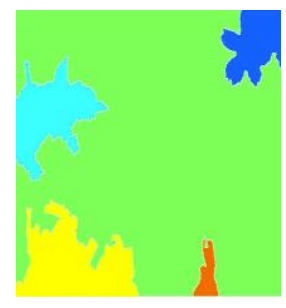

Figure 1.7:(b)

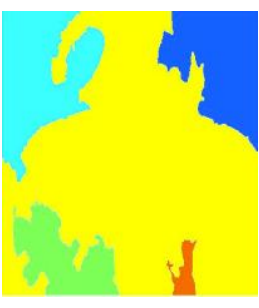

Figure 1.7:(c)

Figure 1.7:(a) Red Channel Watershed

Figure 1.7:(b) Green Channel Watershed Figure 1.7:(c) Blue Channel Watershed

Step 8. Pixel Labeling: Labeling of an image is the operation of assigning a unique value to pixels belonging to the same connected region. Depending on the definition of a "connected region", different results can be obtained.

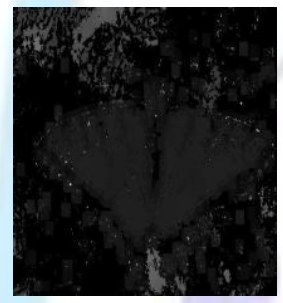

Figure 1.8:(a)

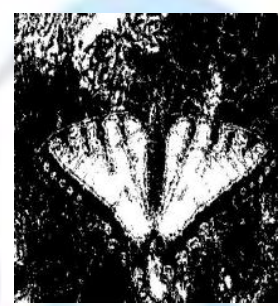

Figure 1.8:(b)

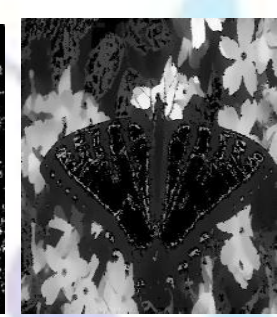

Figure 1.8:(c)

Figure 1.8:(a) Red Channel Segmented Image Figure 1.8:(b) Green Channel Segmented Image Figure 1.8:(c) Blue Channel Segmented Image

Step 9. Final Segmented Image: The Final Gradient image, which is marker, extracted is subjected to Watershed Segmentation. Watershed segmentation produces a more stable segmentation of objects including continuous segmentation boundaries by a concept of producing catchment basin and watershed line.

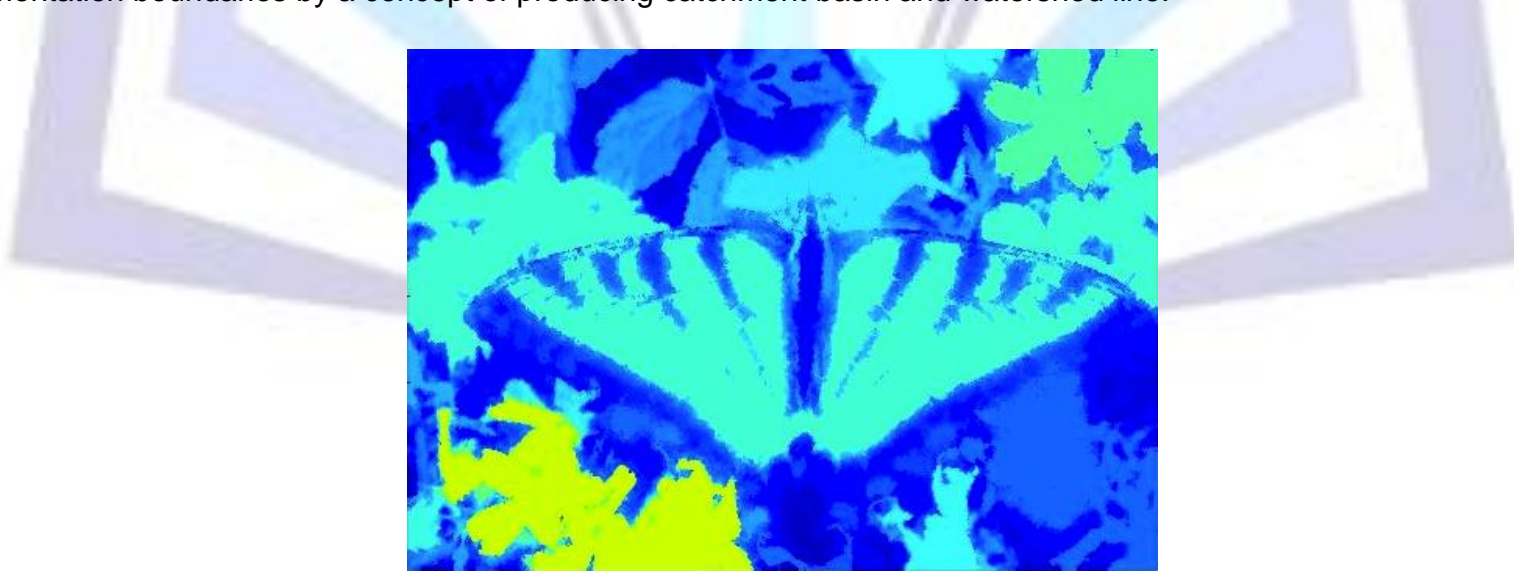

Figure 1.9: Segmented Output

\section{CONCLUSION}

It has been shown that the over-segmentation problem has been ignored in the most of existing work. The noise has also found to be critical issue for image segmentation techniques. Therefore to overcome this problem; we have presented a modified watershed segmentation using Decision based median filtering (DBMF) and Dynamic thresholding (DT). DBMF has the ability to reduce the high density of noise in the image so will provide more efficient results. However DT has also refined segmentation process further. In near future we will extend this work to propose a new technique which will modify the image watershed based segmentation using fuzzy based markers to enhance the results further. 


\section{REFERENCES}

[1] Yuqian Zhao, Jianxin Liu, Huifen Li, and Guiyuan Li,“Improved watershed algorithm for dowels image segmentation," 7th IEEE World Congress on Intelligent Control and Automation, Year 2008. pp. 7644-7648.

[2] Xiaoyan Zhang, Yong Shan, Wei Wei, and Zijian Zhu, "An image segmentation method based on improved watershed algorithm," IEEE International Conference on Computational and Information Sciences (ICCIS), Year 2010,pp.258-261.

[3] Ashwin Kumar and Pradeep Kumar, "A New Framework for Color Image Segmentation Using Watershed Algorithm", Computer Engineering and Intelligent Systems, Vol. 2, No.3 pp.41-46, 2011.

[4] Anju Bala, "An Improved Watershed Image Segmentation Technique using MATLAB," International Journal of Scientific \& Engineering Research Vol.3, No.6, pp.1-4, June 2012.

[5] Yuncai Zhou and Hui Ren,"Segmentation Method for Rock Particles Image Based on Improved Watershed Algorithm," IEEE International Conference on Computer Science \& Service System (CSSS), 2012, pp.347-349.

[6] Jie Chen, Meng Lei, Yao Fan, and Yi Gao,"Research on an improved watershed algorithm to image segmentation," 7th International Conference on Computer Science \& Education (ICCSE), 2012, pp.1917-1919.

[7] Changmin Zhang, Shuaiqi Zhang, Junxia Wu and Shaoxiong Han,"An improved watershed algorithm for color image segmentation," 2012 International Conference on Computer Science and Electronics Engineering (ICCSEE), Year 2012, pp.69-72.

[8] Md.Sharifur Rahman and Md.Rafiqual Islam," Counting objects in an image by marker controlled watershed segmentation and thresholding," 3rd IEEE International on Advance Computing Conference (IACC), 2013, pp.12511256.

[9] Xianwei Han, Yili Fu and Haifeng Zhang,"A fast two-step marker-controlled watershed image segmentation method,"International Conference on Mechatronics and Automation (ICMA), Year 2012, pp.1375-1380.

[10] Boren Li, Mao Pan, and Zixing Wu, "An improved segmentation of high spatial resolution remote sensing image using Marker-based Watershed Algorithm,” 20th IEEE International Conference on Geoinformatics, Year 2012, pp.1-5.

[11] Farheen K. Siddiqui and Vineet Richhariya,"An Efficient Image Segmentation Approach through Enhanced Watershed Algorithm," Computer Engineering and Intelligent Systems, Vol.4, No.6, pp 1-7,2013.

[12] P.P. Acharjya, A. Sinha, S. Sarkar, S. Dey and S. Ghosh,“A New Approach Of Watershed Algorithm Using Distance Transform Applied To Image Segmentation," International Journal of Innovative Research in Computer and Communication Engineering, Vol.1, No.2, pp 185-189, April 2013.

[13] Dibyendu Ghoshal and Pinaki Pratim Acharjya,"Effect of Various Spatial Sharpening Filters on the Performance of the Segmented Images using Watershed Approach based on Image Gradient Magnitude and Direction," International Journal of Computer Applications, Vol. 82, No.6, pp 19-25, November 2013.

[14] Md.Habibur Rahman and Md.Rafiqual Islam,"Segmentation of color image using adaptive thresholding and masking with watershed algorithm," IEEE International Conference onInformatics, Electronics \& Vision (ICIEV), Year 2013, pp.1-6.

[15] Rabul H Laskar, Kalyan Banerjee and Debajit Basak,"Removal of High Density Salt and Pepper Noise from Color Images through Variable Window Size," IEEE International Conference on Circuits, Power and Computing Technologies, 2013, pp. 1132-1136.

[16] S. Beucher, F. Meyer,"The morphological approach to segmentation: The water- shed transformation, Mathematical Morphology in Image processing, Marcel Dekker Inc",New York, pp.433-481, 1993.

[17] M.Sonka, V.Hlavac, and R.Boyle, Image Processing, Analysis, and Machine Vision, PWS Publishing, 1999.

[18] "The Berkeley Segmentation Dataset Benchmark," http://www.eecs.berkeley.edu/Research/Projects/CS/vision/bsds/

[19] “Image Gradient,"[Last Published:] Dec. 2013, [online]. Available: http//www.wikipedia.com

[20] “Image Segmentation,"[Last Published:] Dec. 2013, [online]. Available: http//www.wikipedia.com

[21] Shyam Lal, Sanjeev Kumar and Mahesh Chandra, "Removal of High Density Salt and Pepper Noise Through Super Mean Filter for Natural Images," International Journal of Computer Science, Vol.9, No.3, pp.303-309, May 2012.

[22] V.R. Vijay Kumar, S. Manikandan, D. Ebenezer, P. T. Vanathi, and P. Kanagasabapathy,"High Density Impulse noise Removal in Color Images Using Median Controlled Adaptive Recursive Weighted Median Filter," IAENG International Journal of Computer Science, Vol.34, No.1, August 2007.

[23] S.Varshney Sapna, Navin Rajpal and Ravindar Purwar,"Comparative study of image segmentation techniques and object matching using segmentation," IEEE International Conference on Methods and Models in Computer Science, Year 2009 pp.1-6.

[24] Nguyen Tran Lan Anh, Young-Chul Kim and Guee Sang Lee," Morphological gradient applied to new active contour 
model for color image segmentation," ACM 6th International Conference on Ubiquitous Information Management and Communication, Year 2012.

[25] Shuangqun $\mathrm{Li}$, Jiucheng $\mathrm{Xu}$, Jinyu Ren and Tianhe $\mathrm{Xu}$,"A color image segmentation algorithm by integrating watershed with region merging," Springer Berlin Heidelberg Rough Sets and Knowledge Technology, Year 2012, pp. 167-173. 